\title{
Change of Direction Assessment Following Anterior Cruciate Ligament Reconstruction: A Review of Current Practice and Considerations to Enhance Practical Application
}

\author{
Joao Beleboni Marques ${ }^{1} \cdot$ Darren James Paul ${ }^{1} \cdot$ Phil Graham-Smith $^{2} \cdot$ Paul James Read $^{1}$
}

Published online: 17 September 2019

(c) The Author(s) 2019

\begin{abstract}
Change of direction $(\mathrm{CoD})$ has been indicated as a key mechanism in the occurrence of anterior cruciate ligament (ACL) injury during invasion sports. Despite these associations, assessments of knee function in athletic populations at the time of return to sport following ACL reconstruction (ACLr) have often focused on strength and single-leg hop tests, with a paucity of evidence to describe the $\mathrm{CoD}$ characteristics. Therefore, the aim of this narrative review was to describe the movement strategies exhibited following ACLr during CoD tasks and to critically analyze the range of tests that have been used. Specifically, we examined their ability to identify between-limb deficits and individuals who display a heightened risk of secondary injury and/or reductions in their level of pre-injury performance. MEDLINE, PubMed and SPORT Discuss databases were used and 13 articles were identified that met the inclusion criteria. Examination of the available literature indicates that current field-based practices are not representative of relevant sport demands and are unable to effectively assess knee function following ACLr. Laboratory-based studies have identified residual deficits and altered movement strategies at the time of return to sport, and this in part may be related to risk of re-injury. However, these assessments exhibit inherent limitations and are not practically viable for monitoring progress during rehabilitation. Consequently, alternative solutions that are more-aligned with the multitude of factors occurring during CoD maneuvers in chaotic sports environments are warranted to allow practitioners to 'bridge the gap' between the laboratory and the sports field/court. This approach may facilitate a more informed decision-making process with the end goal being, a heightened 'return to performance' and a lower risk of re-injury.
\end{abstract}

\section{Introduction}

The anterior cruciate ligament (ACL) is commonly injured during sports participation, particularly those involving jumping, pivoting, cutting or change of direction $(\mathrm{CoD})$ maneuvers [32]. ACL-deficiency is suggested to cause knee instability and changes in motor control strategy (i.e., proprioception, postural control, muscle strength, movement and recruitment pattern) [14]. Thus, athletes who wish to continue in hard cutting and pivoting sports after ACL injury are often advised to undergo ACL reconstruction (ACLr) to re-establish mechanical knee stability [52]. However, statistics show that 35\% of

Joao Beleboni Marques

joao.marques@aspetar.com

1 Aspetar, Orthopaedic and Sports Medicine Hospital, Sports City Street, Al Waab, Beverly Hills Garden 14, Villa 30, P.O. Box 29222, Doha, Qatar

2 Aspire Academy, Sports City Street, P.O. Box 29222, Doha, Qatar athletes after ACLr do not return to pre-injury sports level within 2 years [4]. Moreover, up to $20 \%$ of those returning to sport in the first year from surgery experience a second ACL rupture [85].

Evidence suggests criterion-based rehabilitation and return to sport (RTS) progressions should be evaluated with appropriate tests of impairments, activities, participation and contextual factors and combined with a minimum 9-month time criterion [9]. The 2016 consensus on RTS [5] outlines five recommendations to guide the choice of RTS tests: (1) use a group of tests (test battery). (2) Choose open tasks (less controlled) over closed tasks (more controlled) where possible. (3) Include tests with reactive decision-making elements. (4) Assess psychological readiness to return to sport. (5) Monitor internal and external workload. However, a recent scoping review indicates that published research on RTS often does not mirror the 2016 consensus statement [9]. The efficacy of current 'evidenced based' practice in the area of return to sport assessment has also been questioned, with athletes passing specified criteria reducing the risk of subsequent graft rupture 


\section{Key points}

CoD tasks have been recognized as a key mechanism of non-contact ACL injury. However, there is currently a lack of research examining CoD tests as a means to assess knee function following ACLr and associations with secondary injuries or a return to pre-injury levels of performance.

Existing literature offers a combination of field and laboratory-based CoD tests. Laboratory-based assessments appear to be more sensitive in their ability to identify alterations in movement mechanics following ACLr but they also lack ecological validity and are currently not practically viable to systematically assess knee function during the rehabilitation process.

Practitioners are encouraged to develop practically viable solutions to bridge the gap between laboratory and sports environment, while considering relevant conditions (e.g., planned vs. unplanned, fatigue state) and constraints (e.g., cutting angle, approach velocity). This approach can enhance the RTS decision.

by $60 \%$, but this was combined with $235 \%$ increased risk of contralateral graft rupture [84]. Thus, exploration of other factors that contribute to reductions in overall ACL re-injury risk is warranted.

CoD has been recognized as a mechanism of non-contact ACL injury [53, 64, 82]; however, there is a distinct lack of research pertaining to COD performance as component of return to sport testing and the utility of these assessments to identify associations with secondary injuries or a return to pre-injury levels of competition and performance. The aim of this narrative review is to examine the available literature pertaining to CoD assessment following ACLr to determine their suitability as a readiness to RTS tool. In addition, key considerations are provided to aid in the design of CoD assessment, in an attempt to optimize their use as part of the end stage rehabilitation and return to sport process.

\section{Methodology}

The lead author conducted computer searches of MEDLINE, PubMed and SPORT Discuss electronic databases for studies pertaining to CoD assessments following ACLr published between 1970 and April 2109. The search strategy chose to combine specific terms with the words 'change of direction' and 'anterior cruciate ligament reconstruction' to ensure relevant articles were extracted. This included: 'change of direction test following anterior cruciate ligament reconstruction', 'change of direction assessment following anterior cruciate ligament reconstruction', 'functional assessments following anterior cruciate ligament reconstruction', 'assessment of knee function following anterior cruciate ligament reconstruction', 'return to sport criteria following anterior cruciate ligament reconstruction', 'knee mechanics during change of direction following anterior cruciate ligament reconstruction'. Studies were deemed relevant after scanning the title and abstract and where subsequent access to the full text was available. The reference lists of each study were also examined to ensure no further articles were omitted from the search process. Inclusion in this review was based on:

(a) Study design: original articles that describe CoD characteristics of patients following ACLr published in English language. Systematic reviews, conference abstracts, case studies, narrative reviews and non-peer reviewed studies were excluded.

(b) Participants: male and female patients at any age or activity level (e.g., athletes or non-athletes) who had undergone primary ACLr with an autograft (i.e., hamstring or bone-patellar tendon-bone) or allograft surgery techniques. Studies that have not reported ACLr as the primary procedure performed were excluded.

(c) Outcomes: any measure or index adopted to describe CoD performance and/or movement characteristics following ACLr.

(d) Time: all postsurgical time frames were included.

\section{Change of Direction as a Risk Factor for ACL Injury}

A change of direction is characterized as the "skills and abilities needed to change movement direction, velocity, or modes" [22]. It is a highly demanding task recognized as a key mechanism of non-contact ACL injury [53, 64, 82]. External knee valgus moment, internal rotation moment and knee flexion angles are considered to be movement patterns that are causative factors of ACL injuries during CoD [49, 55, 64]. While this may be the mechanical profile of how an ACL injury occurs, there are also many additional factors relating to the execution of CoD tasks that contribute to increased risk. For example, evidence shows ACL injury incidence may be increased during; competition compared to training [79], the latter stages of competition [6], unanticipated reactive tasks [46], close proximity to an opponent [12], defending [12], as well as attacking [53], visually distracted situations [26], when 
carrying equipment [18] and/or dual tasks where attention is divided [3]. This denotes that the occurrence of an ACL injury is complex and there are many possible nuances that may result in this catastrophic outcome.

\section{Change of Direction Following ACL Reconstruction}

Change of Direction tests have been less commonly applied during ACL assessment protocols compared to other tests including strength and hop testing [9]. This approach provides some indication of an individual physical capacity; however, these tests alone may provide an incomplete evaluation of an athlete functional capability following ACLr [57]. Existing literature has used a combination of field and lab-based tests to assess knee function following ACLr and to determine readiness to RTS (Table 1).

\subsection{Field-Based Assessments}

Three field-based testing protocols have commonly been adopted [34, 38, 39, 43, 48]. These includes the shuttle run, co-contraction, and carioca tests. The shuttle run test (Fig. 1a) has been suggested to reproduce acceleration and deceleration forces that are common in sports activities [43]; the co-contraction test (Fig. 1b) imparts rotational forces on the knee that cause tibial translation and are mostly controlled by thigh musculature; while the carioca test (Fig. 1c) reproduces the pivot-shift phenomenon in the ACL-deficient knee when subjects move laterally with a cross-over step [43].

Kong and colleagues [43] reported moderate to high test re-test reliability $(r=0.51-0.74)$ of all three of the aforementioned CoD assessments (shuttle run, co-contraction and carioca tests) as well as their relationships with isokinetic strength $(r=0.45-0.52)$ and one leg hop for distance tests $(r=0.59-0.75)$ on both the involved and uninvolved limbs. These data are supported by earlier research which showed similar relationships $(r=0.46-0.53, p<0.05)$ between these tests and quadriceps isokinetic strength 6 months after ACLr [38]. However, it should be acknowledged that the isokinetic testing modes used were concentric only [38, 43], and eccentric modes of contraction (for both the quadriceps and hamstrings) may be more relevant to CoD tasks. Other studies advocated that these tests were sensitive to distinguish athletes' readiness to return to sports activity $[34,48]$. Keays et al. [38] observed that ACLr patients improved their time in the shuttle run, co-contraction and carioca tests by 10,17 , and $23 \%$, respectively, at 6-month post-surgery compared to pre-surgery values. According to Lephart et al. [48] and Jang et al. [34], better performance was found amongst shuttle run, co-contraction and carioca tests for the patients that returned to sports within 1-2 years after ACLr compared to those who did not return.

Most recently, Kyritsis et al. [44] used the $T$ test with a performance-based cut-off pass criterion of $<11 \mathrm{~s}$ as part of a RTS testing battery to identify athletes' who sustained a secondary ACL rupture [44]. No associations were shown between performance on this test and risk of future ACL injury; however, athletes who did not pass the entire battery of tests (including isokinetic strength and 3 single hop tests), had a four times greater risk of sustaining a second ACL injury compared to those who met the full criteria [44]. Myer et al. [56] proposed a modified version of the $T$ test (Fig. 1d) to examine if differences between involved and uninvolved limbs (by providing alternate directions of travel) could be identified in athletes following ACLr. Similarly, this approach was unable to identify differences between limbs, with the authors indicating the length of the test and the inherent repeated bilateral nature of the task with multiple contacts on each foot mask deficits of the involved lower extremity.

\subsection{Laboratory Assessments}

Stearns and Pollard [78] compared the kinematics and joint kinetics of female soccer players with a history of ACL reconstruction vs. healthy matched controls during a $45^{\circ}$ sidestep cutting maneuver. The assessment was performed after players were cleared to return to full sports participation ( $>9$-month post ACLr). The authors found that players with a history of ACLr displayed increased knee abduction angles and knee adductor moments compared with healthy players. This finding is consistent with the proposed mechanism of increased loading of the ACL and hence, may predispose athletes to a greater risk of re-injury. Using the same $\mathrm{CoD}$ task $\left(45^{\circ}\right.$ sidestep cutting), Pollard et al. [67] examined lower extremity coupling variability in female soccer players 12-month post ACLr. The authors considered this approach as an alternative to examine joint interactions and the variability of these interactions (i.e., neuromuscular control) during a complex movement such as $\mathrm{CoD}$. It has been reported that ACLr players exhibited increased lower extremity variability as compared with the healthy counterparts in the following couplings: hip rotation/knee abduction-adduction, hip flexion-extension/ knee abduction-adduction, knee abduction-adduction/knee flexion-extension, and knee abduction-adduction/knee rotation, suggesting a compromised neuromuscular control as a result of ACLr. This impairment in controlling joint movements during $\mathrm{CoD}$ may predispose the players to re-injury upon RTS participation given the higher environmental demands of the sport. Earlier work by Tibone and Antich [80] reported that a cutting index (defined by the authors as the product of the medial-lateral, anterior-posterior, and 


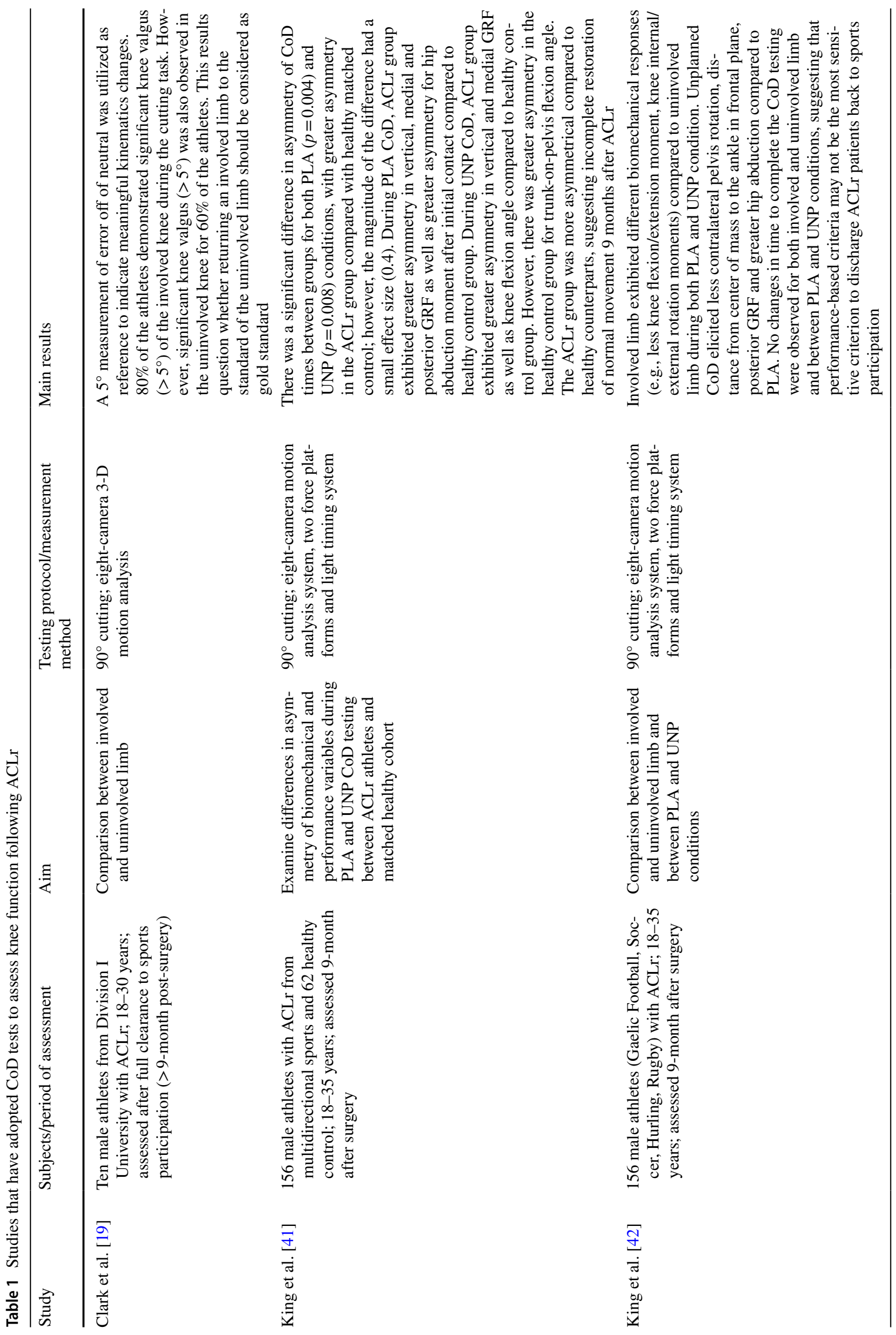




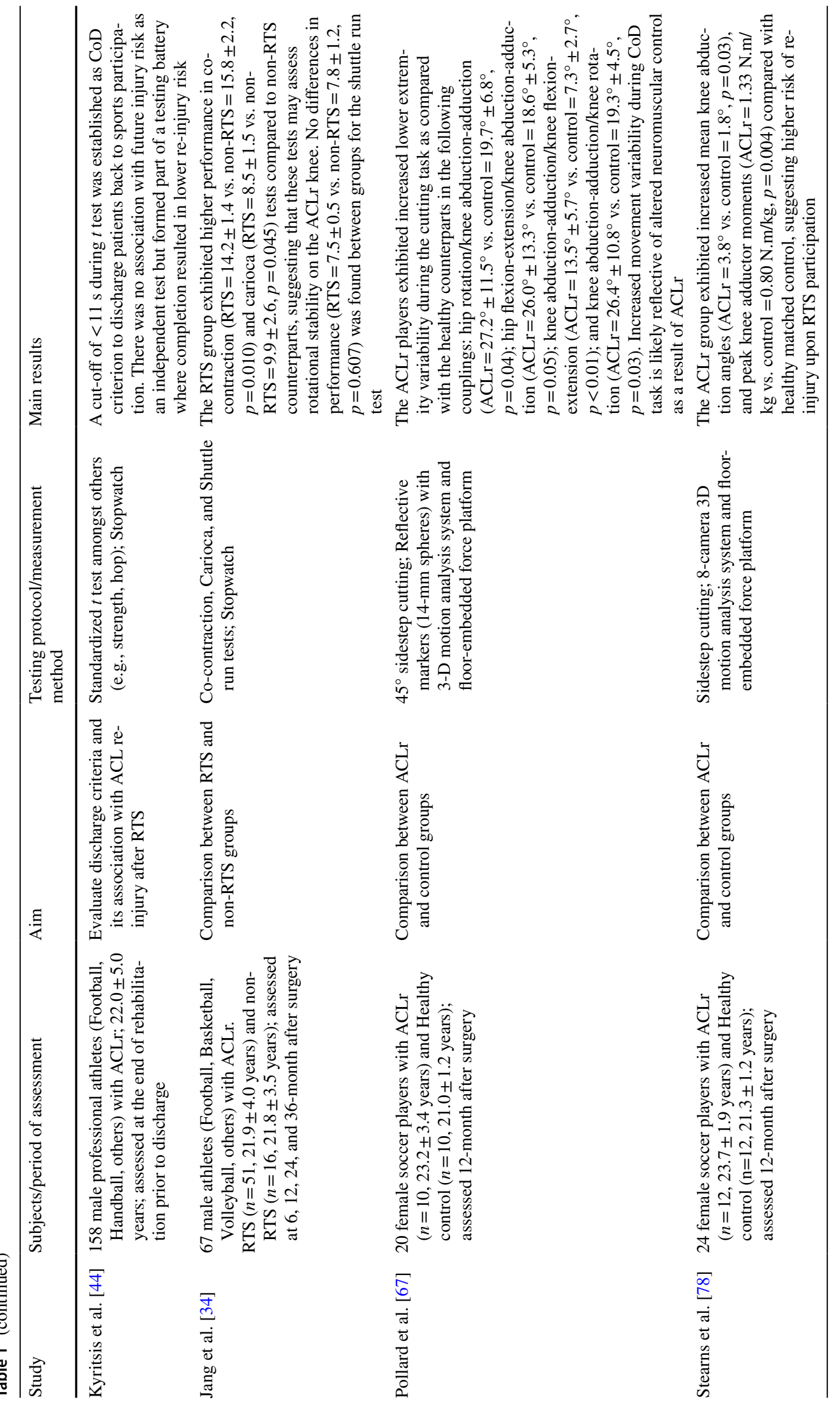




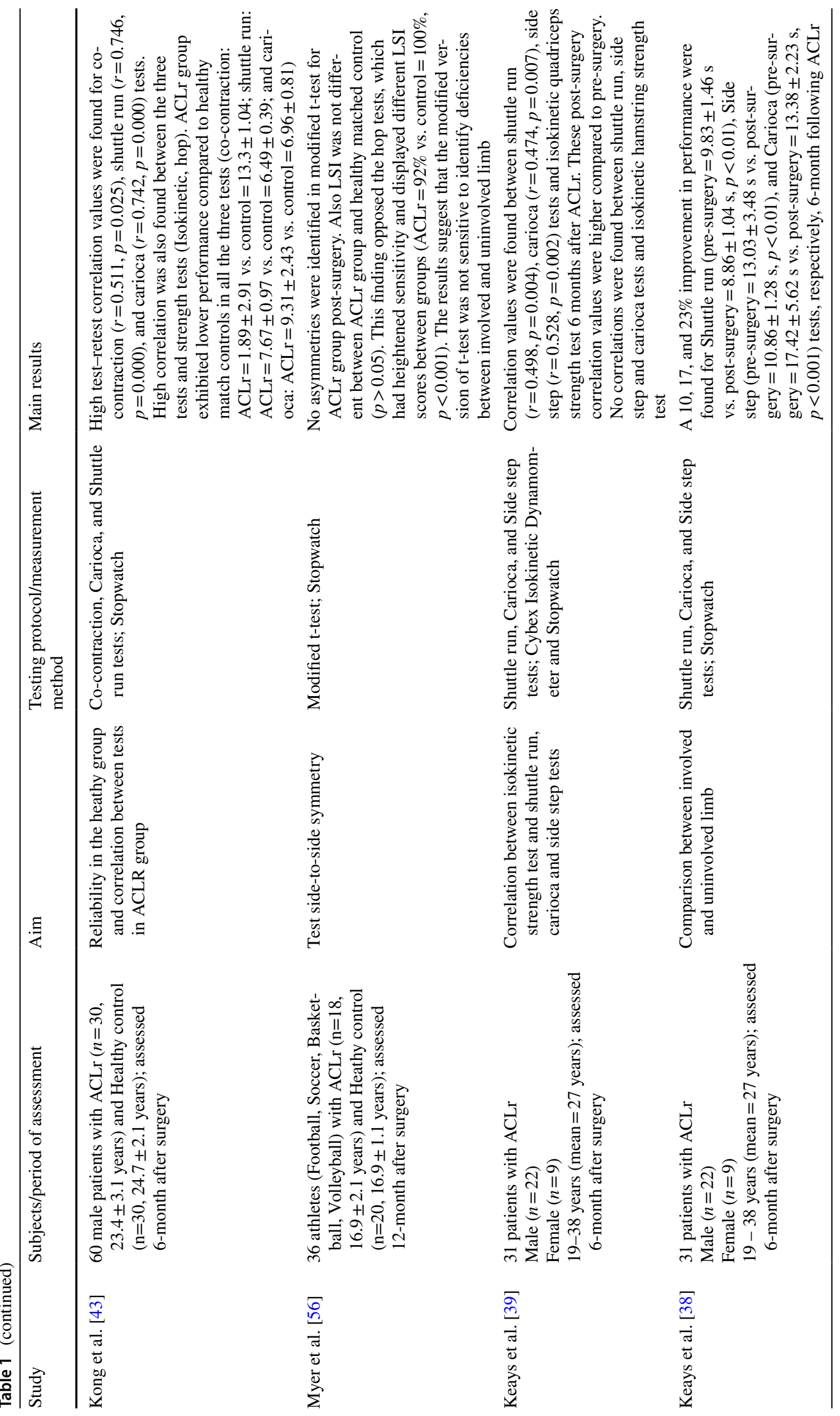


vertical forces and the angle of cut, divided by the product of the approach time and the time spent on the force plate) of a cross cutting task was normalized 2 years after ACLr.

Recently, King et al. [41] examined the performance and biomechanics of a $90^{\circ}$ cutting task in both planned and unplanned (light stimuli) conditions. They tested 156 athletes from different sports background (e.g., Gaelic football, soccer, rugby) 9 months after ACLr. The authors showed different biomechanical responses through the kinetic chain between involved and uninvolved limbs during both $\mathrm{CoD}$ conditions (planned and unplanned) despite no differences in $\mathrm{CoD}$ performance time. The involved limb displayed compensatory mechanisms; specifically, less knee flexion and less knee extension moment in the sagittal plane, and lower knee valgus moment, ankle external rotation moment and knee internal rotation angle and external rotation moment in the frontal/transverse planes. The authors also reported biomechanical differences between planned and unplanned CoDs in variables that have been previously associated with the mechanism of ACL injury (i.e., less contralateral pelvis rotation, distance from center of mass to the ankle in frontal plane, posterior GRF and greater hip abduction) during unplanned cutting. However, similar biomechanical differences have been identified between planned and unplanned $\mathrm{CoD}$ tasks in non-injured athletes [7]. Therefore, it is unknown whether the biomechanical differences found by King et al. [41] were due to deficits following ACLr or to the nature of the task constraints.

In a later analysis, King et al. [42] reported the differences in magnitude of asymmetry of biomechanical and performance variables between the ACLr group and healthy matched controls. They found a significant difference in asymmetry of CoD times between groups for both CoD conditions (planned and unplanned), with greater asymmetry in the ACLr group compared with athletes with no history of ACL injury; however, small magnitude of the difference was reported. For instance, the ACLr group exhibited greater asymmetry in vertical, medial and posterior ground reaction force (GRF) as well as greater asymmetry for hip abduction moment after initial contact compared to control group during planned $\mathrm{CoD}$. For the CoD task under unplanned condition, the ACLr group exhibited greater asymmetry in vertical and medial GRF as well as knee flexion angle compared to healthy matched control. However, there was greater asymmetry in the healthy control group for trunk-on-pelvis flexion angle. These findings suggested that the ACLr group was more asymmetrical compared to healthy counterparts, indicating an incomplete restoration of normal movement 9 months after ACLr.

Using the same CoD task ( $90^{\circ}$ cutting), Clark [19] examined frontal plane knee kinematics in university athletes that have fully returned to sports participation following ACLr. 
(a)

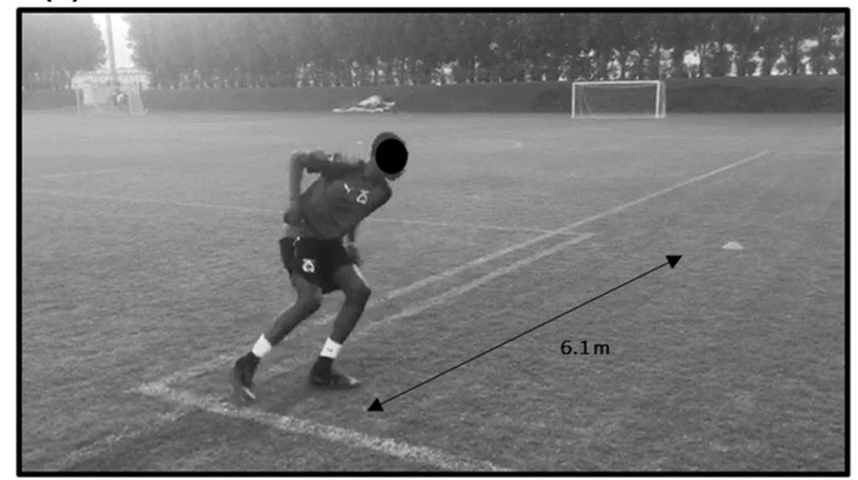

(c)

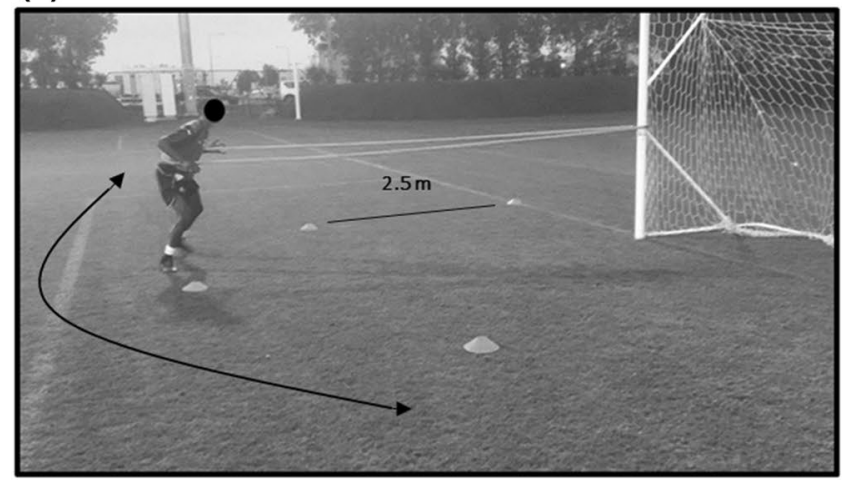

(b)

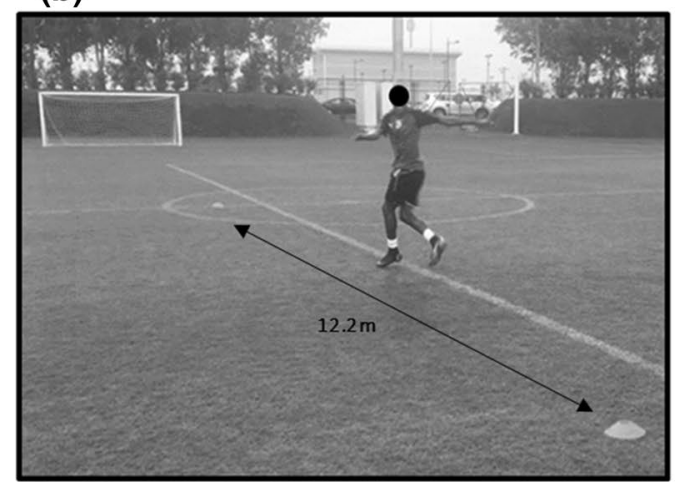

(d)

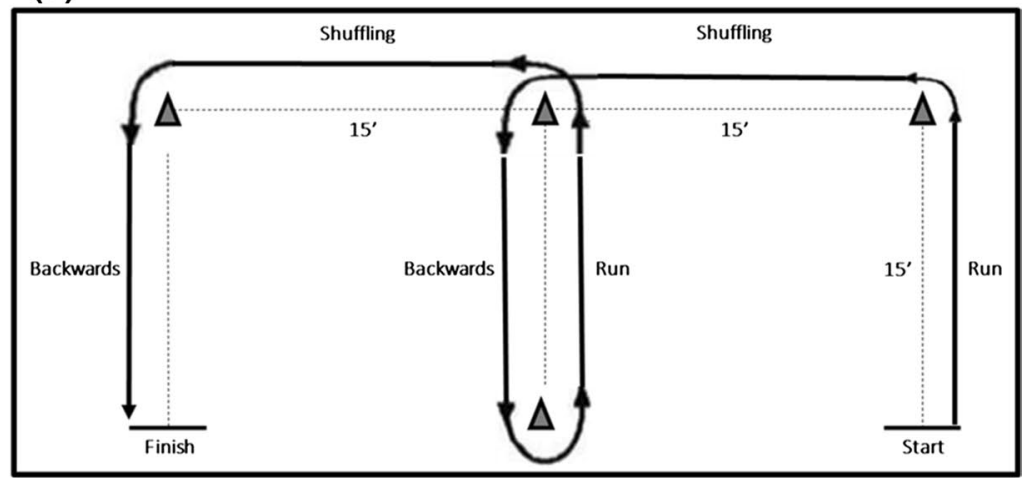

Fig. 1 a-d Illustration of the functional performance tests used to assess knee function following ACLR. a Shuttle run test (adapted from Jang et al. [34]). The athlete performs four lengths of $6.1 \mathrm{~m}$ each to complete $24.4 \mathrm{~m}$ in the shortest amount of time possible, reversing direction after the completion of each length; $\mathbf{b}$ carioca test (adapted from Jang et al. [34]). Using an alternating crossover step, the subject moves laterally to the right $12.2 \mathrm{~m}$, then reverses direction to return

The authors found that 8 out of 10 athletes (80\%) exhibited $\geq 5^{\circ}$ (established as meaningful index value) of knee valgus angle on the involved limb during the CoD task. Interestingly, the authors also reported that $60 \%$ of the uninvolved lower extremities demonstrated a substantial valgus angle as well, suggesting that asymmetries may be inherent to the demands of the CoD task independently of the limb examined. These findings question whether returning an involved limb to the standard of the uninvolved limb should be considered as gold standard metric to discharge patients back to sports participation.

\subsection{Critical Overview of CoD Assessment Used Following ACLr}

In the aforementioned literature, field-based studies have adopted different assessment methods that vary from no changes in direction (e.g., co-contraction, carioca), to 2 (e.g., shuttle run) or 6 directional changes (e.g., agility $t$ test, modified $t$ test). Such variation would elicit different to the starting position; c co-contraction test (adapted from Jang et al. [34]). The patient moves in a side step or shuffle fashion around the periphery of a $2.5 \mathrm{~m}$ radius semicircle. The test is complete when five semicircle lengths have been performed; $\mathbf{d}$ modified $t$ test (adapted from Myer et al. [56]). The test requires a combination of $15 \mathrm{ft}$ of forward running, shuffling and backwards movement to the left side and right sides

energetic requirement and diverse responses of the neuromuscular system; thus, these tests are likely independent from one another [13]. The co-contraction, carioca and shuttle run tests have been recommended by previous studies to inform RTS decision-making [34, 38, 39, 43, 48]. However, it is important to critically appraise their use as CoD tests. Specifically, the movement patterns displayed bears little to no resemblance of how an athlete would move in a sport-specific setting, particularly during cutting and turning maneuvers. CoD at high speed requires an individual to rapidly decelerate to change their state of momentum, and then re-direct their body towards the intended direction of travel prior to re-accelerating with minimal time loss [73]. From these tests, it would appear that the shuttle run test most closely resembles the components of a CoD assessment. However, studies that have adopted this testing protocol have only used completion time (i.e., performance) as a metric to evaluate athletes' readiness which does not examine the strategies used to execute each CoD. It should be noted that during this type of task (i.e., $180^{\circ}$ turn), the 
large majority of the time (70\%) is influenced by linear speed with a substantially lower proportion (30\%) spent during the turning phase [60], and this may mask the actual CoD performance of an athlete [62]. As a result, this approach may not be adequate to identify deficits in movement, particularly between involved and uninvolved limbs.

A method which has been developed to overcome these limitations and isolate the CoD aspect of the task is the CoD deficit [61]. This measure is easily obtained by performing a linear sprint matching the same distance shown during the test and then subtracting this value from the total CoD time (e.g., CoD deficit $=5-0-5$ time $-10 \mathrm{~m}$ sprint time) and may more accurately delineate individuals with better $\mathrm{CoD}$ speed. However, this method has yet to be validated for use in athletes following ACLr. In addition, evaluating the entry and exit velocity may be of interest to more clearly elucidate how the direction change is performed, further removing the effect of confounding factors such as linear speed [62].

In a final point of consideration is that no differences in performance (time to complete the task) scores were frequently observed across the broad range of running based testing protocols in-spite of biomechanical deficits. While laboratory assessments provide some diagnostic value and are useful and help us to better understand the mechanics of $\mathrm{CoD}$, this approach is time consuming, laborious and lacking ecological validity. Practically, sports teams may not have access to these resources and/or may not have the funds to access them. Therefore, consideration of a wider range of contextual factors is needed to develop protocols which are more sensitive to identify deficits in task execution. In addition, the increase in use of micro portable technology [58] may be a more practically viable and cost-effective approach but further research is needed to examine their utility for the assessment of $\mathrm{CoD}$ in athletes return to sport following ACLr.

\section{Assessment Considerations}

\subsection{Competitive vs. Non-competitive}

The essence of all sports is competition. Movements that occur in a laboratory environment will likely be different from movements in real game situations. Performing testing in the laboratory environment is easier to control experimentally but does not consider the presence of opponents and teammates $[45,55]$. While this area has not been examined in athletes following ACLr, exploration may offer an insight into the strategies applied in different sporting contexts that more closely resemble the environments in which they compete and excel. For instance, basketball players were shown to have significantly better reaction time performing against a competitor than by themselves $(690.6 \pm 83.8 \mathrm{~ms}$ vs. $805.8 \pm 101.1 \mathrm{~ms}$, respectively) [89]. Such findings may infer that patients/athletes may not perform 'maximally' during traditional testing when performed alone and the task constraints and test stimuli do not allow elite performers to best utilize their heightened perceptual-cognitive abilities. The inclusion of an opponent/competitor may offer a more appropriate stimulus during which to examine both the athlete's performance and mechanics used. However, this approach is more susceptible to reduced task reliability; thus, further research is warranted to develop a range of CoD assessment modes using human stimuli and examine their ability to provide reproducible and reliable results that can be used to help monitor the athletes return to sport. In addition, virtual reality simulation may provide a suitable competitive environment with a range of scenarios, offering some element of control in a chaotic environment to improve measurement consistency. However, this again requires further investigation, and practically viability should be at the forefront of future developments in this area.

\subsection{Planned vs. Unplanned}

Rarely in match play do rapid direction changes take place in optimal conditions where athletes have time to select the appropriate movement strategy. Risk factors for ACL injury are also higher during unplanned $\mathrm{CoD}$ maneuvers due to the greater external load and mechanical stress placed at the knee joint [7]. Planned CoDs that have been used previously in the assessment of CoD following ACLr (i.e., carioca, shuttle, co contraction, $T$ test, cutting) without temporal constraints may afford sufficient time for the adoption of a 'safer' and more optimal movement execution. For example, the support foot placement strategy (i.e., more medial to the pelvic midline) prior to initial contact of the push-off foot to initiate the direction change, thus lowering the mechanical stress on the knee [47]. Performing an unplanned task can be in response to either generic (react against 2 dimension planned and unplanned light-based directional arrows) and quasi-realistic (react against 1 or 2 defenders' scenarios in a 3-dimension video projection) external stimuli. However, the generic unplanned condition (i.e., reacting to a light system) utilized during the $\mathrm{CoD}$ test does not provide an ecologically valid stimulus. It is important to highlight as well that this type of generic (light) stimulus does not resemble those that frequently are present in sport to distinguish between elite and sub-elite performers [73]. Highperformance athletes will use their "game knowledge" and anticipate situations based on phase of play sequences and then react to the kinematic cues displayed by their opponents $[1,2,25]$. Therefore, using tests that involve generic cues (such as light stimulus) are likely to be limited in their ability to assess transferrable sports specific abilities of athletes which require integration of perception-action coupling, and decision-making to effectively execute a CoD task. 
This lack of realistic scenario may limit our ability to fully understand the impact of a true un-planned 'agility' action on ACL injury risk and this point is further highlighted by recent research.

\subsection{Fatigued vs. Non-fatigued}

Change of direction assessments are typically conducted when athletes are in a fully recovered state. In most sports, fatigue and agility tasks rarely exist independent of one another; thus, their combined manifestation may present as a worst case scenario in terms of injury risk. Studies have attempted to examine fatigue-induced changes in lower extremity mechanics and their association with ACL injury risk following prolonged activities. For instance, Zebis et al. [88], found that muscle fatigue induced by a simulated handball game elicited alterations in neuromuscular responses. A large reduction in ACLagonist muscle (i.e., hamstring) activity has been observed during a side cutting movement following a preceding fatigue protocol (requested participants to perform sidestep and crossover maneuvers, low- to high-intensity running and sprinting intermittently during $50 \mathrm{~min}$ ), decreasing dynamic knee stability at initial contact and this might expose athletes to higher risk of ACL injury [87]. Savage et al. [69] also found larger knee joint angles and knee extension and internal rotation moments during a sidestep cutting task following a prolonged running protocol that simulated a game-like activity (e.g., walk, jog, fast run and maximal sprint). The impact of fatigue on movement pattern has not been exclusively identified through prolonged activities. According to Cortes et al. [17], a short-term (e.g., 6-min) fatigue testing protocol can be sufficient to induce several biomechanical changes in the lower extremity (i.e., decreased knee and hip flexion and increased knee internal rotation moments) during an unplanned sidestep cutting. Although the available evidence does not indicate a clear fatigue mechanism, it is apparent that neuromuscular control is altered when athletes are exposed to either competition [30] and/or protocols designed to elicit fatigue [17]. As a result, it may be relevant to implement CoD testing following short-term (e.g., repeated sprints) or long-term (e.g., simulated game activity) fatigue testing protocols to assess ACLr patients' readiness. This approach allows practitioners to identify the ability of the patient to maintain a safer movement pattern during $\mathrm{CoD}$ while fatigued.

\section{Assessment Characteristics: Task and Environment Constraints}

\subsection{Cutting Angle}

The magnitude of the load placed on the knee joint will be largely determined by the $\mathrm{CoD}$ angle required to perform the test [77]. A $45^{\circ}$ cutting task has commonly been used to assess knee function following $\operatorname{ACLr}[78,80]$ yet there is a heightened injury risk (i.e., knee valgus moment) increase at sharper cutting angles such as $90^{\circ}, 135^{\circ}$ and $180^{\circ}$ turning when compared to $45^{\circ}$ (Fig. 2) [77]. If approach velocity and stopping distance remain consistent across different cutting/turning angles, sharper $\mathrm{CoD}$ angles $\left(\geq 90^{\circ}\right)$ require an athlete to momentarily stop (to reach zero velocity) and brake harder. This increases the task demands as higher ground reaction forces are produced in shorter durations which in turn increases external loading on the knee joint and potential increase risk of injury [51]. In addition to changes in joint loading, the direction of force application during a CoD test is also highly dependent of the cutting angle. Sigward et al. [71] showed that the vertical, posterior and lateral ground reaction forces were $21 \%, 87 \%$ and $228 \%$ greater, respectively, during $110^{\circ}$ cutting task in comparison with a $45^{\circ}$ angle for both male and female soccer players. These data indicate that greater posterior and laterally directed forces are present for sharper $\mathrm{CoD}$ angles.

\subsection{Approach Velocity}

CoD mechanics have also been shown to vary according to the approach velocity during the task, whereby athletes will self-organize their approach speed and entry velocity dependent upon the angle [23]. While not the case in all incidences, it appears non-contact ACL injuries occur more frequently during CoDs performed from higher approach velocities in multidirectional sports [53, 64]. Variables associated with ACL injury (i.e., knee flexion angles, knee valgus loading) increases during $45^{\circ}, 60^{\circ}$ and $135^{\circ}$ cutting tasks from faster running velocities ( 4 and $5 \mathrm{~m} \mathrm{~s}^{-1}$ ) compared to slower velocities $\left(2\right.$ and $3 \mathrm{~m} \mathrm{~s}^{-1}$ ) $[40,58,82]$. Thus, when interpreting the available literature, practitioners should be cognizant of these differences and how they affect joint loading. Athletes returning to sport following ACLr may also intentionally reduce their approach velocity if they perceive the demands of the CoD task are too great relative to their current capacity to decelerate effectively. Therefore, practitioners need to understand the influence and impact of cutting angle and approach velocity on join loading and $\mathrm{CoD}$ task demands in order to develop the most appropriate assessment protocols to assess knee function at different stages of rehabilitation following ACLr. In addition, allowing an athlete to self-select their approach and entry speed during testing (while potentially increasing the variability) will enhance the ecological validity and provide information as to their $\mathrm{CoD}$ strategy which can be monitored during rehabilitation to determine changes in performance and readiness to return to sport. 


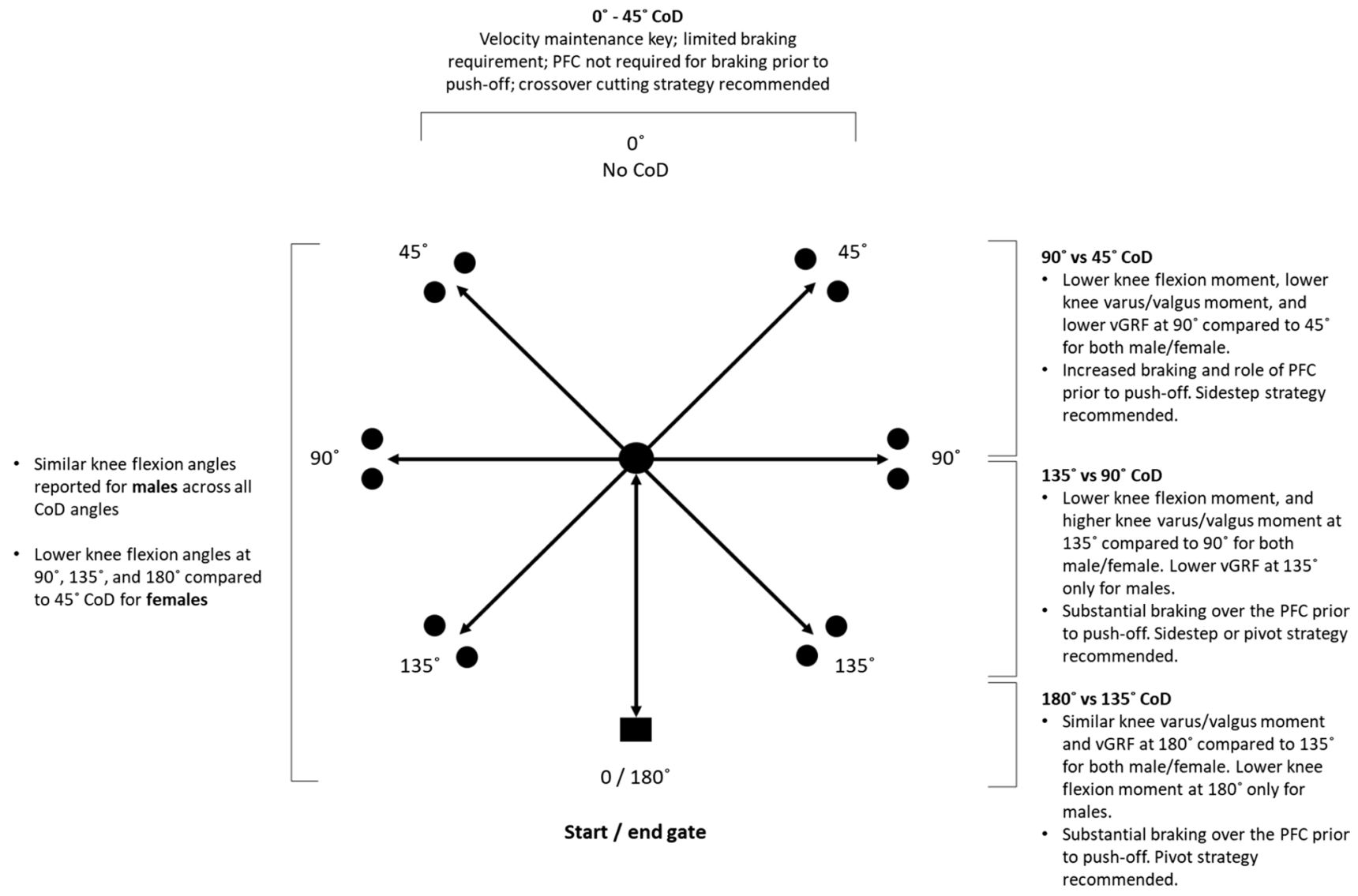

Fig. 2 Kinetic and kinematic characteristics for males and females across different CoD angles (adapted from Dos'Santos et al. [23]; Schreurs et al. [77])

\subsection{Technique}

$\mathrm{CoD}$ technique (kinematics) and the resultant load (kinetics) appear to be dependent on the task demands (e.g., angle, speed and mode) [24]. Athletes typically use three primary CoD techniques: the side-step, crossover cut, and split-step which display differences in execution and resultant mechanics [24]. For example, side-step has been considered as a key technique to feint an opponent, particularly during situations that required sharper $\mathrm{CoD}$; however, it generates larger knee valgus and internal rotation moments compared to crossover cut and split-step techniques, suggesting higher risk of ACL injury [24]. The crossover cut technique on the other hand is commonly adopted by athletes when shallow $\mathrm{CoD}$ under high approach velocity is required to evade a defensive line or to pass by an opponent. Contrarily to side-step technique, crossover cut generates higher knee varus moment and loads the lateral component of the knee [24]. Finally, split-step can generate greater lateral velocity compared to side-step and crossover and hence, opponents find it as a difficult technique to be anticipated. Despite the lower knee abduction load displayed during split-step, this technique elicits longer ground contact time to be executed and as a result, it has been considered as a slower strategy for CoD compared to side-step and crossover techniques [24].

Biomechanical and technique differences have been also reported during extreme $\mathrm{CoD}$ techniques such as $180^{\circ}$ turn compared to shallower cutting angles with athletes adopting different strategies due to the task constraints. Cortes et al. [16] showed that lower knee flexion $\left(-41.2 \pm 8.8^{\circ}\right.$ vs. $\left.-53.9 \pm 9.4^{\circ}\right)$ and a higher valgus angle $\left(-7.6 \pm 10.1^{\circ}\right.$ vs. $\left.-2.9 \pm 10.0^{\circ}\right)$ was present during $180^{\circ} \mathrm{CoD}$ task vs. the sidestep $\left(45^{\circ}\right)$ and, respectively, at the point of maximum vertical ground reaction force. The $180^{\circ} \mathrm{CoD}$ task also had higher peak posterior ground reaction force than the drop-jump $(0.8 \pm 0.3$ multiples of body weight vs. $0.3 \pm 0.06$ multiples of body weight $)$ and side-step cutting $(0.3 \pm 0.1$ multiples of body weight). In addition, higher internal varus moments were indicated in the $180^{\circ} \mathrm{CoD}$ task $(0.72 \pm 0.3 \mathrm{~N} \mathrm{~m} / \mathrm{kg} \mathrm{m})$ as opposed to the drop-jump $(0.14 \pm 0.07 \mathrm{~N} \mathrm{~m} / \mathrm{kg} \mathrm{m})$ and side-step $(0.17 \pm 0.5 \mathrm{~N} \mathrm{~m} / \mathrm{kg} \mathrm{m})$ at peak stance.

According to Condello et al. [15], a more rounded shape strategy has been observed when athletes decrease contact time during the final step of a $60^{\circ}$ cutting task. Team sport athletes usually adopt a sharper cutting strategy to effectively evade an opponent during competition. Thus, decreasing the 
contact time during the final step may be also detrimental for sports performance. The management of this performance-injury conflict is key to prepare athletes to RTS participation following ACLr. Practitioners should therefore, consider such important information to adopt adequate training strategies during the rehabilitation program.

\subsection{Visual Disturbance}

Research has shown that experimentally visually cued temporal constraints can affect whole-body kinematics and knee loading during athletic activities such as cutting [3]. During side-cut conditions while attending to a ball, internal knee adductor moment was $20 \%$ greater $(p=0.03)$ and peak knee flexion angle was $4^{\circ}$ larger $(p<0.01)$, compared to without the ball. Bjornaraa et al. [10] examined whether subjects with ACLr display different displacement, velocity, and time to peak ground reaction force during cutting activities than healthy subjects and whether visual disruption alters these variables. Knee displacement was significantly less for ACLr than non-dominant. Knee velocity was also significantly slower for ACLr vs. non-dominant limb with longer time to reach peak GRF. The authors reported a lack of vision resulted in reduced absolute velocity and displacement, especially for subjects with a history of ACL reconstruction; thus, suggesting the use of a new or altered 'default' motor program. Lieberman and Hoffman [50] observed that subjects with more experience in a task respond with less difference in movement patterns when vision is disrupted. Therefore, one could argue that environments should be as realistic and context-specific as possible when evaluating the ability to RTS. In addition, repeated exposures to these environments should form an important component of end stage rehabilitation to more fully prepare athletes for the demands of their sport.

\subsection{Dual Cognitive Task}

During $\mathrm{CoD}$ tasks performed in competitive situations the cognitive load is increased since the athlete must allocate attentional resources to manipulate the ball and must also be aware of the location of their opponents, teammates and the goal and this in part may contribute to the higher risk of ACL injury during such movements [3, 74]. Negahban et al. [59] examined the effects of attention demands on postural control in ACLr patients who had return to sport $>12$ months after surgery. Patients were required to perform a single-leg stance on a balance board under both singleand dual-task conditions in four dynamic balance tests. The authors showed that ACLr patients displayed higher contact frequency and longer contact time compared to healthy match controls. But more interestingly, ACLr patients showed higher contact frequency and longer contact time during dual-task compared to single-task conditions [59]. These findings highlight that concurrent execution of both postural and cognitive tasks led to performance deterioration of postural stability measures rather than cognitive measures. This suggests that the maintenance of standing balance is more attention demanding for ACLr patients than healthy matched controls. Therefore, it could be inferred that subtle alterations to testing protocols such as having participants attending to a ball [26], a defender [55], a simulated teammate [3], or simultaneous execution of a cognitive task [59] may have prominent effect on an athletes' movement during CoD. As a result, the attentional demands of a task need to be carefully considered if the goal is to develop/implement an ecologically valid testing protocol. It is likely that practitioners may gain additional insight into the mechanics that contribute to non-contact ACL injury if they incorporate testing protocols that are reflective of the cognitive demands of the sports environment; however, due to the paucity of data and likely reductions in experimental control, this requires further investigation.

\subsection{Double Stimulation}

Current COD test are broadly reflective of discrete aspects of athlete movement. Indeed, most COD assessments are either planned with one or few $\mathrm{CoD}$ or basic reactive decision responses (unplanned). This is very different to the complex decision-making process of one, or several, additional actions performed during matches. For example, this may involve two closely spaced movements (stimuli) by an attacking player performing a fake ball pass $[31,75]$. The basis of the feint is the double-stimulation paradigm, where the reaction to the first of two closely spaced stimuli is normal, but the reaction to the second is delayed by more than that which would have occurred had it been presented alone [76]. Current $\mathrm{CoD}$ assessment methods are unable to examine these aspects. Achieving suitable control with acceptable test re-test reliability of such factors which occur in a chaotic environment is a challenging and possibly unrealistic ideal. Therefore, practitioners are encouraged to explore ways in which athletes can be exposed to these stimuli in training, with tasks clearly designed with different levels of contextual interference that are reflective of the athletes' stage of rehabilitation and level of skill challenge required.

\section{Interpreting the Results}

\subsection{Task Completion Time vs. Movement Execution}

Time to complete the task has often been the sole marker for $\mathrm{CoD}$ assessment. Recently, King et al. [41] highlighted 
the limitations of solely using time when they identified biomechanical deficits when cutting using the involved vs. uninvolved limb during a $90^{\circ}$ cutting task despite no differences in performance time. In addition, Kyritsis et al. [44] used a cut-off of 'pass score' of $<11 \mathrm{~s}$ during a $T$ test protocol as one of the criterion to discharge professional athletes back to sports participation. Munro et al. [54] reported mean performance values of $10.7 \mathrm{~s}$ for the same testing protocol performed by recreational university athletes who would be expected to have lower physical capacities. It is the opinion of the authors that $\mathrm{CoD}$ assessments should always have a task performance component but this should not be the only criteria due to the need to examine potential deficits in lower extremity biomechanics which may contribute to a greater risk of re-injury [78]. Using total time solely to measure $\mathrm{CoD}$ is not sufficient to identify important qualitative information (e.g., trunk position, foot placement, center of mass height, knee angles, arm actions and visual focus) presented by an athlete while executing the CoD movement. In addition, practitioners are advised to utilize performance cut-off 'pass scores' that represent the high performances required by elite athletes returning to professional sport if that is the intended destination. Finally, wherever possible, general cut-off 'pass scores' should be replaced by individual pre-injury performance data to make decisions relative to the individual (considering differences in strength, speed, CoD ability, etc.). Applying the same absolute score to all athletes could be too conservative/demanding for faster and slower athletes, respectively, highlighting the importance of collecting base-line data on all players as part of a regular screening/monitoring program.

\subsection{The Importance of Deceleration}

Deceleration is a fundamental component of multidirectional speed to allow athletes to effectively change their state of momentum. Rapid deceleration (stop type activities) are a consideration for ACL injury due to an increase in anterior tibial shear force and anterior tibial translation [70]. Despite deceleration is present in all sprinting and CoD tasks, they have often been investigated individually. Peel et al. [66] examined lower extremity biomechanics between both decelerating and $45^{\circ}$ cutting tasks as well as the relationship between peak anterior shear force (ASF) and peak knee abduction moments (KAM) during both decelerating and cutting tasks. It has been found that the CoD task exhibited significantly higher initial contact knee abduction angles $(p=0.032, d=0.62)$, higher KAM $(p \leq 0.001, d=2.84)$ and larger ASF $(p=0.001, d=1.42)$ compared to the decelerating task. This suggests that CoD induces higher mechanical stress at the knee joint and hence, an increased risk of ACL injury compared to decelerating task. The authors also reported a positive relationship $(p=0.67)$ for ASF during both decelerating and CoD tasks. However, a negative relationship ( $p=-0.21)$ was found between both tasks for KAM, suggesting that decelerating and cutting are independent of one another. In addition, it has been observed a negative correlation $(p=-0.43)$ between ASF and KAM during both decelerating and CoD tasks. While peak ASF increases, peak KAM decreases, indicating that these two variables are autonomous [66]. Due to these distinct differences, it could be suggested that measurement of deceleration as an isolated construct (without the $\mathrm{CoD}$ component) may be an appropriate assessment which can be used at an earlier stage of onpitch/court rehabilitation to examine an individual's ability to effectively apply braking forces as a pre-cursor to CoD.

In support of the need to examine an athlete's approach to deceleration, the role of the preparation steps prior to turning or cutting should be considered. Higher cutting angles (i.e., $\geq 60^{\circ}$ ) require greater reductions in velocity to change the athletes state of momentum [27-29]. Greater braking forces during the penultimate foot contact ensure that athletes can maintain a higher entry velocity (as they can brake later) which results in faster CoD speed, and reduce the ground reaction force on the turning limb during the plant step [36]. This has important connotations as non-contact ACL injuries often occur on the planted limb during a sudden deceleration prior to a change in direction [8]. Maintenance of foot alignment in the direction of travel during the penultimate step ensures the quadriceps are in an advantageous position. This technical model is more effective and potentially 'safer', whereby, the production of the highest magnitude of braking forces required to effectively decelerate takes place in the penultimate, as opposed to the turn step, when the foot has already laterally rotated and is in a more "vulnerable position'. Thus, it may be prudent to examine kinematics and loading characteristics during the penultimate step to more fully understand the athlete's deceleration strategy. Currently, methods to assess these qualities on the field/ court are sparse; thus, further research is warranted using practically viable testing protocols.

\subsection{Limb Symmetry}

As previously indicated, biomechanical differences between involved (ACLr limb) and uninvolved limb were found during both planned and unplanned CoD tasks despite no differences in task performance [41]. Myer et al. [56] also did not identify performance differences when assessing the $T$ test performance favoring either the involved or un-involved limb. These data suggest that athletes develop compensatory movement strategies to complete the task with similar performance levels. This finding questions whether returning an involved limb to the standard of the uninvolved limb should be considered as the gold standard during tasks such as CoD. It is also unclear if the current level of performance on the uninvolved limb is representative of their pre-injury 
capacity [86]. Furthermore, Thomas et al. [81] reported that athletes adopt different braking strategies between dominant and non-dominant limbs during a $180^{\circ}$ turn, whereby, a greater magnitude of horizontal braking forces is placed on the penultimate step by the non-dominant limb when turning with the dominant limb. Conversely, a greater emphasis is placed on the final foot contact when turning with non-dominant leg [81]. Thus, limb symmetry is just one component of the return to sport puzzle and assessment of movement characteristics during $\mathrm{CoD}$ should be considered alongside the demands of the sport, estimated pre-injury capacity, preferred limb dominance and potential risk for re-injury.

\section{Practical Applications}

Based on the aforementioned considerations, a CoD assessment model is illustrated in Figure 3. While not designed to be prescriptive, the information provides examples of a progression and regression sequence of four-fundamental aspects discussed within the current review (i.e., angle, speed, fatigue and task constraint). The aim of this approach is to illustrate how key test conditions/constraints can be manipulated for effective prescription of $\mathrm{CoD}$ assessment (and training) at different stages of rehabilitation following ACLr. This model may help practitioners to establish appropriate CoD testing protocols in agreement with the load athletes are able to tolerate based on their level of function and stage of rehabilitation.

For example, exposing athletes who are returning to sport following ACLr to sharper CoD angles requires an abducted lower extremity position, leading to greater external loading on the knee joint and subsequent higher risk of re-injury [51]. However, this approach is necessary to create a sufficient lateral foot plant distance to apply effective propulsive forces and execute the task with maximal performance $[33,35]$. Since sharper cutting angles and faster changes in direction are determinants for successful performance in multidirectional and invasion sports $[11,68]$, practitioners

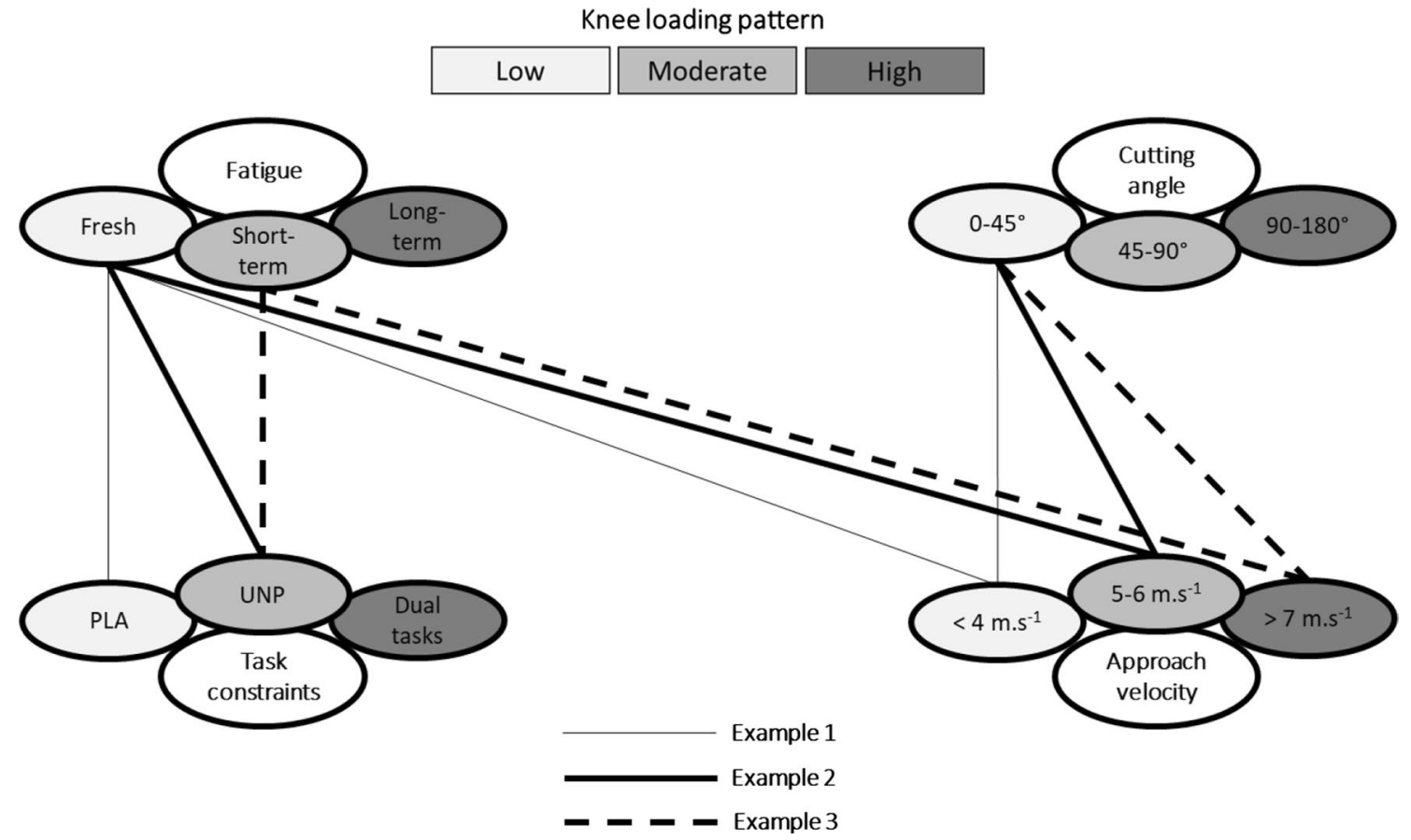

Fig. 3 The model displays an illustrative example of a CoD assessment progression sequence to support practitioners in setting up effective $\mathrm{CoD}$ test prescription to assess knee function following ACLr. The figure demonstrates how key testing conditions/constraints can be manipulated to increase knee loading progressively during a $45^{\circ} \mathrm{CoD}$ task across the rehabilitation process. Example 1 requires low approach velocity $\left(<4 \mathrm{~m} \mathrm{~s}^{-1}\right)$, in a non-fatigue state, under planned conditions. This approach induces low stress at the knee joint and can be performed at the beginning of the functional phase of rehabilitation in order to allow practitioners to identify potential technical issues (i.e., foot placement) while executing the CoD task. Example 2 is a progression and includes higher approach velocity
(5-6 $\mathrm{m} \mathrm{s}^{-1}$ ) and a constraint during the CoD task (e.g., responding to an external stimulus). Finally, example 3 represents an assessment design that induces higher stress at the knee joint and hence, its implementation may be more appropriate during an advanced phase of the rehabilitation process (i.e., sport-specific phase). It requires high approach velocity $\left(>7 \mathrm{~m} \mathrm{~s}^{-1}\right)$, followed by a short-term fatigue testing protocol (e.g., repeated sprints), under unplanned conditions. This approach may allow practitioners to identify progressions in movement execution in relation to previous assessments, as well as the ability of the patient to maintain appropriate mechanics during the $\mathrm{CoD}$ task while fatigued 
should be aware of this performance-injury conflict. A lack of exposure to these more extreme changes of direction may leave the athletes underprepared for the demands of match play, leading to an increased risk of re-injury. Conversely, shallower cutting angles display lower joint loading and may be more appropriate for those in the earlier phases of their rehabilitation.

Another example of how these considerations can also be shown using the concept of approach velocity. Low approach velocities will reduce knee joint loading; however, it will not prepare them for sport-specific scenarios. In professional sport, athletes are unlikely to sacrifice performance at the expense of reduced knee joint loading [23]. Therefore, this performance-injury conflict should once again be managed during the rehabilitation process. Prior to exposing ACLr patients to tasks at maximal approach velocities during a CoD test, practitioners should ensure that patients display appropriate CoD mechanics $[20,21,35,45]$ and the requisite physical capacities to tolerate the associative knee joint loading [37, 63, 65, 72, 83]. Furthermore, approach velocities (and distances) should be gradually increased as the athlete progress and moves closer to return to sport. Specifically, monitoring of approach velocity during maximal CoD tasks could also be a practically viable approach and may be linked to confidence as well as physical and technical competence. Furthermore, integration of other factors discussed within this review, such as double stimulation and visual disturbance can be utilized where appropriate to create heightened contextual interference in the later stages of rehabilitation as a means of increasing the cognitive overload of CoD tasks.

\section{Conclusion}

CoD assessments have not been widely implemented by practitioners as a criterion measure to inform RTS decisionmaking. Furthermore, current field-based practices do not appear in agreement with relevant sport demands, or effective in their assessment of knee function following ACLr. Laboratory-based studies have shown residual deficits in movement mechanics in athletes at the time of return to sport, and this in part may be related to risk of re-injury. However, these assessments have shown several limitations and are likely not practically viable for monitoring progress during rehabilitation.

In this review we have detailed a number of factors for the development of future practice in the area of CoD assessment following ACLr and provided suggestions for how these can be incorporated. Fundamentally, individual risk factors for the athlete and their sport should be examined to determine their state of readiness to RTS. In doing so, it is essential that clinicians understand the different layers of CoD assessment; most importantly, the conditions/constraints under which they are performed. Analysis of movement mechanics is also an important focus; as total time alone is likely insufficient to accurately characterize performance decrements. To do this, practically viable solutions for on-pitch/court measurement are now needed to allow coaches to 'bridge the gap' between the laboratory and the sports environment. This approach may facilitate a more informed decision-making process with the end goal being, a 'return to performance' with a lower risk of re-injury.

\section{Compliance with Ethical Standards}

Funding Open Access funding provided by the Qatar National Library. Also, no financial support was received for the preparation of this manuscript.

Conflict of Interest Joao Marques, Darren Paul, Phil Graham-Smith, and Paul Read declare that they have no conflict of interest.

Open Access This article is distributed under the terms of the Creative Commons Attribution 4.0 International License (http://creativeco mmons.org/licenses/by/4.0/), which permits unrestricted use, distribution, and reproduction in any medium, provided you give appropriate credit to the original author(s) and the source, provide a link to the Creative Commons license, and indicate if changes were made.

\section{References}

1. Abernethy B, Russell DG. Expert—novice difference in an applied selective attention task. J Sport Psychol. 1987;9:326-45.

2. Ageberg E, Thomeé R, Neeter C, Silbernagel KG, Roos EM. Muscle strength and functional performance in patients with anterior cruciate ligament injury treated with training and surgical reconstruction or training only: a two to five-year follow up. Arthritis Rheum. 2008;59:1773-9.

3. Almonroeder TG, Kernozek T, Cobb S, Slavens B, Wang J, Huddleston W. Divided attention during cutting influences lower extremity mechanics in female athletes. Sports Biomech. 2017;13:1-13

4. Ardern CL, Taylor NF, Feller JA, Webster KE. Fifty-five per cent return to competitive sport following anterior cruciate ligament reconstruction surgery: an updated systematic review and metaanalysis including aspects of physical functioning and contextual factors. Br J Sports Med. 2014;48:1543-52.

5. Ardern CL, Glasgow P, Schneiders A, Witvrouw E, Clarsen B, et al. 2016 Consensus statement on return to sport from the First World Congress in Sports Physical Therapy, Bern. Br J Sports Med. 2016;50:1-12.

6. Beaulieu ML, Wojtys EM, Ashton-Miller JA. Risk of anterior cruciate ligament fatigue failure is increased by limited internal femoral rotation during in vitro repeated pivot landings. Am J Sports Med. 2015;43:2233-41.

7. Besier TF, Lloyd DG, Ackland TR, Cochrane JL. Anticipatory effects on knee joint loading during running and cutting maneuvers. Med Sci Sports Exerc. 2001;33:1176-81.

8. Boden BP, Dean GS, Feagin JA Jr, Garrett WE Jr. Mechanisms of anterior cruciate ligament injury. Orthopedics. 2000;23:573-8. 
9. Burgi CR, Peters S, Ardern CL, Magill JR, Gomez CD, Sylvain J, Reiman MP. Which criteria are used to clear patients to return to sport after primary ACL reconstruction? A scoping review. $\mathrm{Br}$ J Sports Med. 2019;53:1-10.

10. Bjornaraa J, Di Fabio RP. Knee kinematics following ACL reconstruction in females; the effect of vision on performance during cutting task. Int J Sports Phys Ther. 2011;6:271-84.

11. Bloomfield J, Polman R, O'Donoghue P. Turning movements performed during FA Premier League soccer matches. J Sports Sci Med. 2007;6:9-10.

12. Brophy RH, Stepan JG, Silvers HJ, Mandelbaum BR. Defending puts the anterior cruciate ligament at risk during soccer: a genderbased analysis. Sports Health. 2015;7:244-9.

13. Brughelli M, Cronin J, Levin G, Chaouachi A. Understanding change of direction ability in sport: a review of resistance training studies. Sports Med. 2008;38:1045-63.

14. Courtney CA, Rine RM. Central somatosensory changes associated with improved dynamic balance in subjects with ACL deficiency. Gait Posture. 2006;24:190-5.

15. Condello G, Kernozek TW, Tessitori A, Foster C. Biomechanical analysis of a change-of-direction task in college soccer players. Int J Sports Physiol Perform. 2016;11:96-101.

16. Cortes N, Onate J, Van Lunen B. Pivot task increases frontal plane loading compared with sidestep and drop-jump. J Sports Sci. 2011;29:83-92.

17. Cortes N, Quammen D, Lucci S, Greska E, Onate J. A functional agility short-term fatigue protocol changes lower extremity mechanics. J Sports Sci. 2012;30:797-805.

18. Chaudhari A, Marquand A, Hearn B, Leveille L, Johnson E, Andriacchi T. Sport-dependent variations in arm position during single limb landing affect knee loading: implications for ACL injury. Am J Sports Med. 2005;33:824-30.

19. Clark N, Forshey T, Mulligan I, Kindel C. Knee mechanics during a change of direction movement in division I athletes following full return to sport from anterior cruciate ligament reconstruction. Phys Ther Sport. 2019;35:75-8.

20. Dempsey AR, Lloyd DG, Elliott BC, Steele JR, Munro BJ, Russo KA. The effect of technique change on knee loads during sidestep cutting. Med Sci Sports Exerc. 2007;39:1765-73.

21. Dempsey AR, Lloyd DG, Elliott BC, Steele JR, Munro BJ. Changing sidestep cutting technique reduces knee valgus loading. Am J Sports Med. 2009;37:2194-200.

22. DeWeese B, Nimphius $\mathrm{S}$. Speed and agility program design and technique. In: Triplett NT, Haff GG, editors. Essentials of strength and conditioning. Champaign: Human Kinetics; 2016. p. 521-57.

23. Dos'Santos T, Thomas C, Comfort P, Jones PA. The effect of angle and velocity on change of direction biomechanics: an anglevelocity trade-off. Sports Med. 2018;48:2235-53.

24. Dos'Santos T, McBurnie A, Thomas C, Comfort P, Jones PA. Biomechanical comparison of cutting techniques. A review and practical applications. Strength Cond J. 2019. https://doi.org/10.1519/ ssc. 0000000000000461 .

25. Farrow D, Chivers P, Hardingham C, Sachse S. The effect of video-based perceptual training on the tennis return of serve. Int J Sport Psychol. 1998;29:231-42.

26. Fedie R, Carlstedt K, Willson JD, Kernozek TW. Effect of attending to a ball during a side-cut maneuver on lower extremity biomechanics in male and female athletes. Sports Biomech. 2010;9:165-77.

27. Hader K, Palazzi D, Buchheit M. Change of direction speed in soccer: how much braking is enough? Kinesiology. 2015;47:67-74.

28. Hader K, Mendez-Villanueva A, Palazzi D, Ahmaidi S, Buchheit M. Metabolic power requirement of change of direction speed in young soccer players: not all is what it seems. PloS One. 2016;11:e0149839.
29. Havens KL, Sigward SM. Whole body mechanics differ among running and cutting maneuvers in skilled athletes. Gait Posture. 2014;42:240-5.

30. Hawkins RD, Fuller CW. An examination of the frequency and severity of injuries and incidents at three levels of professional football. Br J Sports Med. 1998;32:326-32.

31. Henry G, Dawson B, Lay B, Young W. Effects of a feint on reactive agility performance. J Sports Sci. 2012;30:787-95.

32. Hewett TE, Myer GD, Ford KR. Preparticipation physical examination using a box drop vertical jump test in young athletes: the effects of puberty and sex. Clin J Sport Med. 2006;16:298-304.

33. Inaba Y, Yoshioka S, Iida Y, Hay DC, Fukashiro S. A biomechanical study of side steps at different distances. J Appl Biomech. 2013;29:336-45.

34. Jang SH, Kim JG, Ha JK, Wang BG, Yang SJ. Functional performance tests as indicators of returning to sports after anterior cruciate ligament reconstruction. Knee. 2014;21:95-101.

35. Jones PA, Herrington LC, Graham-Smith P. Technique determinants of knee joint loads during cutting in female soccer players. Hum Mov Sci. 2015;42:203-11.

36. Jones PA, Herrington L, Graham-Smith P. Braking characteristics during cutting and pivoting in female soccer players. $\mathrm{J}$ Electromyogr Kinesiol. 2016;30:46-54.

37. Jones P, Thomas C, Dos'Santos T, McMahon J, Graham-Smith $\mathrm{P}$. The role of eccentric strength in $180^{\circ}$ turns in female soccer players. Sports (Basel). 2017. https://doi.org/10.3390/sport s5020042.

38. Keays SL, Bullock-Saxon J, Keays AC. Strength and function before and after anterior cruciate ligament reconstruction. Clin Orthop Relat Res. 2000;373:174-83.

39. Keays SL, Bullock-Saxton JE, Newcombe P, Keays AC. The relationship between knee strength and functional stability before and after anterior cruciate ligament reconstruction. J Orthop Res. 2003;21:231-7.

40. Kimura K, Sakurai S. A sidestep cut preparation strategy decreases the external load applied to the knee joint. Int J Sport Health Sci. 2013;11:109-17.

41. King E, Richter C, Franklyn-Miller A, Daniels K, Wadey R, Jackson M, Moran R, Strike S. Biomechanical but not timed performance asymmetries persist between limbs 9 months after ACL reconstruction during planned and unplanned change of direction. J Biomech. 2018;81:93-103.

42. King E, Richter C, Franklyn-Miller A, Wadey R, Moran R, Strike S. Back to normal symmetry? Biomechanical variables remain more asymmetrical than normal during jump and change-of-direction testing 9 months after anterior cruciate ligament reconstruction. Am J Sports Med. 2019;47:1175-85.

43. Kong DH, Yang SJ, Ha JK, Jang SH, Seo JG, Kim JG. Validation of functional performance tests after anterior cruciate ligament reconstruction. Knee Surg Relat Res. 2012;24:40-5.

44. Kyritsis P, Bahr R, Landreau P, Miladi R, Witvrouw E. Likelihood of ACL graft rupture: not meeting six clinical discharge criteria before return to sport is associated with a four times greater risk of rupture. Br J Sports Med. 2016;50:946-51.

45. Kristianslund E, Faul O, Bahr R, Myklebust G, Krosshaug T. Sidestep cutting technique and knee abduction loading: implications for ACL prevention exercises. Br J Sports Med. 2014;48:779-83.

46. Lee MJ, Lloyd DG, Lay BS, Bourke PD, Alderson JA. Effects of different visual stimuli on postures and knee moments during sidestepping. Med Sci Sports Exerc. 2013;45:1740-8.

47. Lee MJ, Lloyd DG, Lay BS, Bourke PD, Alderson JA. Different visual stimuli affect body reorientation strategies during sidestepping. Scand J Med Sci Sports. 2017;27:492-500.

48. Lephart SM, Perrin DH, Fu FH, Gieck JH, McCue FC, Irrgang JJ. Relationship between selected physical characteristics and 
functional capacity in the anterior cruciate ligament-insufficient athlete. J Orthop Sports Phys Ther. 1992;16:174-81.

49. Leppanen M, Pasanen K, Kujala UM, Vasankari T, Kannus P, Ayramo S, Krosshaug T, Bahr R, Avela J, Perttunen J, Parkkari J. Stiff landings are associated with increased ACL injury risk in young female basketball and floorball players. Am J Sports Med. 2017;45:386-93.

50. Lieberman DG, Hoffman JR. Timing of preparatory landing responses as a function of availability of optic flow information. J Electromyogr Kinesiol. 2005;15:120-30.

51. Marshall BM, Franklyn-Miller AD, King EA, Moran KA, Strike S, Falvey A. Biomechanical factors associated with time to complete a change of direction cutting maneuver. J Strength Cond Res. 2014;28:2845-51.

52. Marx RG, Jones EC, Angel M, Wickiewicz TL, Warren RF. Beliefs and attitudes of member of the American Academy of Orthopaedic Surgeons regarding the treatment of anterior cruciate ligament injury. Arthroscopy. 2003;19:762-70.

53. Montgomery C, Blackburn J, Withers D, Tierney G, Moran C, Simms C. Mechanisms of ACL injury in professional rugby union: a systematic video analysis of 36 cases. Br J Sports Med. 2018;52:994-1001.

54. Munro AG, Herrington LC. Between-session reliability of four hop tests and the agility $t$ test. J Strength Cond Res. 2011;25:1470-7.

55. McLean SG, Lipfert SW, Van Den Bogert AJ. Effect of gender and defensive opponent on the biomechanics of sidestep cutting. Med Sci Sports Exerc. 2004;36:1008-16.

56. Myer GD, Schmitt LC, Brent JL, Ford KR, Barber Foss KD, Scherer BJ, Heidt RS Jr, Divine JG, Hewett TE. Utilization of modified NFL combine testing to identify functional deficits in athletes following ACL reconstruction. J Orthop Sports Phys Ther. 2011;41:377-87.

57. Narducci E, Waltz A, Gorski K, Leppla L, Donaldson M. The clinical utility of functional performance tests within one-year post-ACL reconstruction: a systematic review. Int J Sports Phys Ther. 2011;6:333-42.

58. Nedergaard NJ, Kersting U, Lake M. Using accelerometry to quantify deceleration during a high-intensity soccer turning manoeuvre. J Sports Sci. 2014;32:1897-905.

59. Negahban H, Ahmadi P, Salehi R, Mehravar M, Goharpey S. Attentional demands of postural control during single leg stance in patients with anterior cruciate ligament reconstruction. Neurosci Lett. 2013;27:118-23.

60. Nimphius S, Geib G, Spiteri T, Carlisle D. Change of direction deficit measurement in division I american football players. J Aust Strength Cond. 2013;21:115-7.

61. Nimphius S, Callaghan SJ, Spiteri T, Lockie RG. Change of direction deficit: a more isolated measure of change of direction performance than total 505 time. J Strength Cond Res. 2016;11:3024-32.

62. Nimphius S, Callaghan SJ, Bezodis NE, Lockie RG. Change of direction and agility tests: challenging our current measures of performance. Strength Cond J. 2017;40:1-13.

63. Nimphius S. Training change of direction and agility. In: Turner A, Comfort P, editors. Advanced strength and conditioning. Abdingdon: Routledge; 2018. p. 291-308.

64. Olsen OE, Myklebust G, Engebretsen L, Bahr R. Injury mechanisms for anterior cruciate ligament injuries in team handball a systematic video analysis. Am J Sports Med. 2004;32:1002-12.

65. Padua DA, Di Stefano LJ, Hewett TE, Garrett WE, Marshall SW, Golden GM, Shultz SJ, Sigward SM. National athletic trainers' association position statement: prevention of anterior cruciate ligament injury. J Athl Train. 2018;53:5-19.
66. Peel SA, Schroeder LE, Sievert ZA, Weinhandl JT. Comparing anterior cruciate ligament injury risk variables between unanticipated cutting and decelerating tasks. J Appl Biomech. 2019;35:101-6.

67. Pollard CD, Stearns KM, Hayes AT, Heiderscheit BC. Altered lower extremity movement variability in female soccer players during side-step cutting after anterior cruciate ligament reconstruction. Am J Sports Med. 2015;43:460-5.

68. Robinson G, O'Donoghue P, Nielson P. Path changes and injury risk in English FA Premier League soccer. Int J Perform Anal Sport. 2011;11:40-56.

69. Savage RJ, Lay BS, Wills JA, Lloyd DG, Doyle TLA. Prolonged running increases knee moments in sidestepping and cutting manoeuvres in sport. J Sci Med Sports. 2018;21:508-12.

70. Sell TC, Akins JS, Opp AR, Lephart SM. Relationship between tibial acceleration and proximal anterior tibia shear force across increasing jump distance. J Appl Biomech. 2014;30:75-81.

71. Sigward SM, Cesar GM, Havens KL. Predictors of frontal plane knee moments during side-step cutting to 45 and 110 degrees in men and women: implications for anterior cruciate ligament injury. Clin J Sport Med. 2015;25:529-34.

72. Suchomel TJ, Nimphius S, Stone MH. The importance of muscular strength in athletic performance. Sports Med. 2016;46:1419-49.

73. Sheppard JM, Young WB. Agility literature review: classifications, training and testing. J Sports Sci. 2006;24:919-32.

74. Shultz SJ, Schmitz RJ, Benjaminse A, Collins M, Ford K, Kulas AS. ACL research retreat VII: an update on anterior cruciate ligament injury risk factor identification, screening, and prevention. $\mathrm{J}$ Athl Train. 2015;50:1076-93.

75. Schmidt RA, Lee TD. Motor control and learning: a behavioral emphasis. 4th ed. Champaign: Human Kinetics; 2005.

76. Schmidt RA, Wrisberg CA. Motor learning and performance: a situation-based learning approach. 4th ed. Champaign: Human Kinetics; 2008.

77. Schreurs MJ, Benjaminse A, Lemmink KA. Sharper angle, higher risk? The effect of cutting angle on knee mechanics in invasion sport athletes. J Biomech. 2017;63:144-50.

78. Stearns KM, Pollard CD. Abnormal frontal plane knee mechanics during sidestep cutting in female soccer athletes after anterior cruciate ligament reconstruction and return to sport. Am J Sports Med. 2013;41:918-23.

79. Stubbe JH, van Beijsterveldt AMMC, van Knaap S, Stege J, Verhagen EA, van Mechelen W, Backx FJ. Injuries in professional male soccer players in the Netherlands: a prospective cohort study. J Athl Train. 2015;50:211-6.

80. Tibone JE, Antich TJ. A biomechanical analysis of anterior cruciate ligament reconstruction with the patellar tendon. A two year follow up. Am J Sports Med. 1988;16:332-5.

81. Thomas C, Dos'Santos T, Kyriakidou I, Cuthbert M, Fields C, Jones PA. An investigation into the effect of limb preference on knee mechanics and braking strategy during pivoting in female soccer players: An exploratory study. In: The 8th Annual Strength and Conditioning Student Conference, Middlesex University: London, UK; 2017.

82. Vanrenterghem J, Venables E, Pataky T, Robinson MA. The effect of running speed on knee mechanical loading in females during side cutting. J Biomech. 2012;45:2444-9.

83. Weinhandl JT, Earl-Boehm JE, Ebersole KT, Huddleston WE, Armstrong BS, O'connor KM. Reduced hamstring strength increases anterior cruciate ligament loading during anticipated sidestep cutting. Clin Biomech. 2014;29:752-9.

84. Webster KE, Hewett TE. What is the evidence for and validity of return-to-sport testing after anterior cruciate ligament reconstruction surgery? A systematic review and meta-analysis. Sports Med. 2019. https://doi.org/10.1007/s40279-019-01093-x. 
85. Wiggins AJ, Grandhi RK, Schneider DK, Stanfield D, Webster $\mathrm{KE}$, Myer GD. Risk of secondary injury in younger athletes after anterior cruciate ligament reconstruction: a systematic review and meta-analysis. Am J Sports Med. 2016;44:1861-76.

86. Wilk K. We can do better. J Orthop Sport Phys Ther. 2014;44:634-5.

87. Zebis MK, Andersen LL, Bencke J, Kjaer M, Aagaard P. Identification of athletes at future risk of anterior cruciate ligament ruptures by neuromuscular screening. Am J Sports Med. 2009;37:1967-73.
88. Zebis MK, Bencke J, Andersen LL, Alkjaer T, Suetta C, Mortensen P, Kjaer M, Aagaard P. Acute fatigue impairs neuromuscular activity of anterior cruciate ligament-agonist muscles in female team handball players. Scand J Med Sci Sports. 2010;21:833-40.

89. Zemkova E, Vilman T, Kovacikova Z, Hamar D. Reaction time in the agility test under simulated competitive and non-competitive conditions. J Strength Cond Res. 2013;27:3445-9. 